\title{
Piezoelectric power generation from zinc oxide nanowires grown on paper substrate
}

Muhammad Yousuf Soomro, I Hussain, Nargis Bano, Omer Nur and Magnus Willander

\section{Linköping University Post Print}

N.B.: When citing this work, cite the original article.

This is the authors' version of the original publication:

Muhammad Yousuf Soomro, I Hussain, Nargis Bano, Omer Nur and Magnus Willander, Piezoelectric power generation from zinc oxide nanowires grown on paper substrate, 2012, Physica Status Solidi. Rapid Research Letters, (6), 2, 80-82.

http://dx.doi.org/10.1002/pssr.201105519

Copyright: Wiley-VCH Verlag Berlin http://www.wiley-vch.de/publish/en/

Postprint available at: Linköping University Electronic Press http://urn.kb.se/resolve?urn=urn:nbn:se:liu:diva-76026 


\title{
Piezoelectric power generation from zinc oxide nanowires grown on paper substrate
}

\author{
M. Y. Soomro, ${ }^{*}$ I. Hussain, N. Bano, O. Nur, M. Willander \\ Department of Science and Technology, Campus Norrköping, Linköping University, SE-60174 Norrköping, Sweden
}

Keywords Zinc oxide, nanowires, piezogenerator, paper substrate

*Corresponding author: e-mail muhso@itn.liu.se

In this study, we demonstrate piezoelectric power generation from zinc oxide $(\mathrm{ZnO})$ nanowires grown on paper substrate. Vertically aligned $\mathrm{ZnO}$ nanowires are deflected by an atomic force microscopy (AFM) tip in contact mode which generates an output voltage of up to $7 \mathrm{mV}$. Furthermore, the effects of different parameters mainly influencing the magnitude of the output voltage are discussed. We expect that due to its simplicity,

During the past few years, bottom-up approach of nanotechnology has ascertained its enormous influence on our economy, environment and society with a variety of innovative products based on different nanostructures. Nanoscale devices are the main emphasis for future technology which can revolutionize our daily life but the main obstacle is how to provide suitable power to such devices. We cannot use traditional batteries or an external power source for nanodevices because it will make the system size relatively very large. One effective and alternative approach in this regard is to develop nanoscale power generating devices. In this context a nanogenerator has been developed which scavenges energy from the environment by utilizing the coupling between the electrical and the mechanical properties [1]. Intense world-wide research efforts have been devoted to find out the best materials which significantly enhanced the piezoelectric effect capability.

$\mathrm{ZnO}$ is one of the most important multi-functional semiconductors that possess strong piezoelectric effect [2]. $\mathrm{ZnO}$ has one of the highest effective piezoelectric coefficients among the different tetrahedrally coordinated wurtzite compound semiconductors e.g. GaN, CdS, AlN and CdSe. This makes it an interesting electromechanical coupling material for various piezoelectric applications [3-6]. ZnO has a rich family such as nanorods, nanowires, nanobelts and nanotubes and all can be synthesized under this approach represents an important step within the development of nanoscale power generators. It offers a promising alternative powering source for the next generation nanodevices on disposable paper.

specific growth conditions [7]. Recently, various applications based on the piezoelectric properties of the $\mathrm{ZnO}$ nanostructures grown on variety of conventional substrates have been demonstrated like e.g. piezoelectric field effect transistors, the piezoelectric diode, piezoelectric sensors and the piezoelectric nanogenerator [1, 8-10]. The paper used as a substrate can be one of the best alternates for piezoelectric applications due to many advantages over conventional substrates like low-cost, relatively abundant, flexible, light in weight, nonhazardous and fully recyclable. Nanoscale power generator on paper substrate can provide an effective platform for powering nanoscale devices.

In this paper we demonstrate piezoelectric effect and generation of piezo potential from $\mathrm{ZnO}$ nanowires (NWs) grown by the low temperature aqueous chemical growth (ACG) method on paper substrate by using atomic force microscopy. This represents an attractive alternative approach and may open the ways for research and development of nanogenerators on paper substrate which can provides an effective platform for powering nanoscale devices in future. Finally, different parameters affecting the output potential are also discussed.

$\mathrm{ZnO}$ NWs were grown on a piece of clean room paper used as a substrate by the low temperature aqueous chemical growth (ACG) method [11]. Clean room paper provided by VWR International is designed especially for 
cleanroom environments. It consists of cellulose with properties like, lint-free, low particle content to minimize risk of contamination. In order to make an electric contact at the bottom of the $\mathrm{ZnO}$ nanowires a thin layer of silver (Ag) was deposited by thermal evaporation method and is used as a bottom electrode during the experiment. The $\mathrm{Ag}$ coated paper substrate was ultrasonically cleaned with DI water and dried with nitrogen. Then a $\mathrm{ZnO}$ seed layer was spin coated on the Ag coated paper substrate and backed at $100{ }^{0} \mathrm{C}$ for $30 \mathrm{~min}$. To grow $\mathrm{ZnO} \mathrm{NWs}$ zinc nitrate hexahydrate $\left[\mathrm{Zn}\left(\mathrm{NO}_{3}\right)_{2} \cdot 6 \mathrm{H}_{2} \mathrm{O}\right](99.998 \%)$ and hexamethylenetetramine (HMT) $\left(\mathrm{C}_{6} \mathrm{H}_{12} \mathrm{~N}_{4}\right)(99.998 \%)$ having the same molar concentration were mixed in DI water. The precoated substrate was then placed in the solution and heated at $85{ }^{\circ} \mathrm{C}$ for $5 \mathrm{~h}$. After the growth, the sample was washed with DI water and was dried by nitrogen. The $\mathrm{ZnO}$ NWs grown on the paper substrates were found to be vertically aligned and distributed uniformly as shown in the SEM image in Fig. 1.

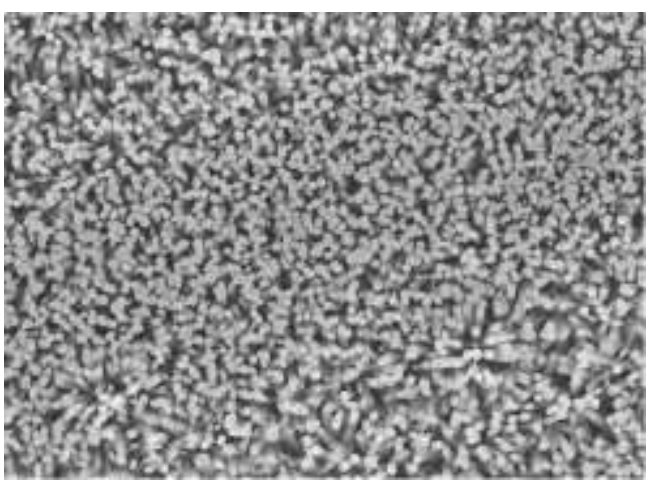

Figure 1 Top view SEM image of the ZnO NWs grown on the paper substrate by the low temperature ACG growth.

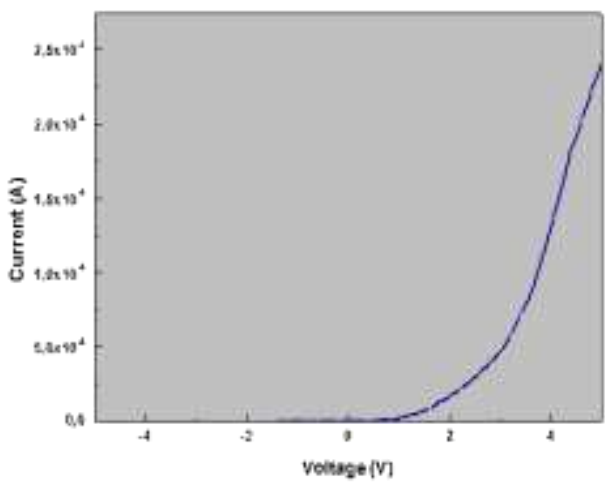

Figure 2 The current-voltage characteristics showing Schottkytype behaviour.

The piezoelectric measurements were performed using contact mode of an atomic force microscopy (AFM) from Digital Instruments Multimode (Nanoscope IIIA controller) having a tetrahedral conductive Si tip coated with platinum $(\mathrm{Pt})$ film. There are dual advantages of using Pt coat- ed tip. First it increases the conductivity of the electrode and secondly it makes a Schottky contact between the $\mathrm{ZnO} \mathrm{NWs}$ and the tip which is critical for the piezoelectric power generation and also plays a major role in the entire process of electron transport and it directs the current to flow from the electrode to the NWs. The currentvoltage (I-V) characteristic shown in Fig. 2 was measured before conducting the piezoelectric experiment. We grounded the Ag bottom electrode by using Ag paste and the tip of the AFM used as Schottky contact during the I-V measurements. When the tip scans over the surface of the sample the output signal voltage was continuously monitored. A resistor is connected between the fixed end of the $\mathrm{ZnO} \mathrm{NW}$ and the AFM tip. During the scanning of the $\mathrm{ZnO} N W s$, a constant force of about $80 \mathrm{nN}$ is sustained between the tip and sample. The tip scans with a speed of 30 $\mu \mathrm{m} / \mathrm{s}$ over the top of the sample surface over an area of about $2 \times 2 \mu \mathrm{m}^{2}$ from right to left. During the scanning process the aligned NWs were bent repeatedly and the bending distance was recorded in the topography image as shown in the Fig. 3.

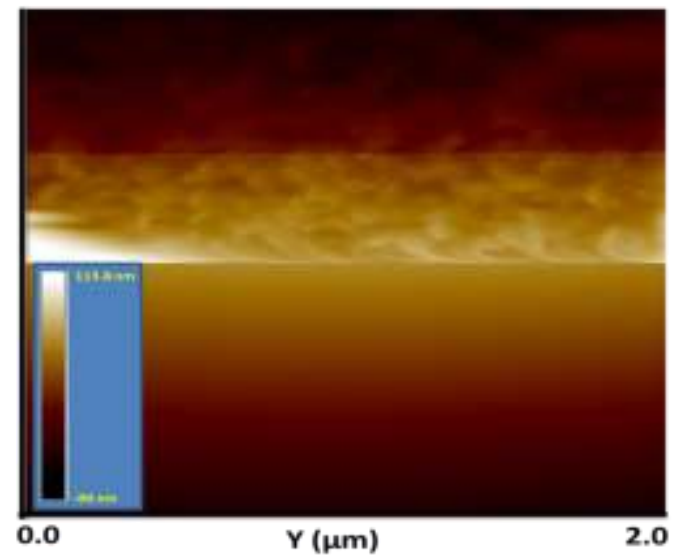

Figure 3 Topography image (feedback signal from the scanner) showing the bending distance of the $\mathrm{ZnO} \mathrm{NW}$ arrays during scanning.

When the AFM tip touches the stretched side of the $\mathrm{ZnO}$ NWs, the Pt-ZnO contact become a reverse biased Schottky interface, because Pt has a work function $(\Phi)$ of $6.1 \mathrm{eV}$ which is greater than the electron affinity $(\chi)$ of $\mathrm{ZnO}$ $(\sim 4.35 \mathrm{eV})$. This interface serves as a gate for blocking the back flow of electrons and only negligible current would flow across the interface. This is the process that creates, separates and preserves the piezoelectric potential. On the other hand, when the AFM tip touches the compressed side of the $\mathrm{ZnO}$ NWs, the metal tip-ZnO interface will become forward biased Schottky contact resulting in an electrical output voltage drop as shown in the Fig. 4. This is the discharging process and flow of electrons takes place on the expenses of the piezoelectric potential from the tip into the NWs. Three dimensional (3D) plot of the output voltage corresponding to the feedback signal from the 
scanner is shown in Fig. 5. The output voltage can be in the range of a fraction of a volt with some sharp peaks ranging from 4 to $11 \mathrm{mV}$. The output peaks are corresponding to the maximum deflection of the NWs.

Previously our group reported piezoelectric power generation with an output potential of about $5 \mathrm{mV}$ from $\mathrm{ZnO}$ nanowires grown on different conventional semiconductor substrates like SiC and Si [12]. Now through this approach the magnitude of the output voltage is around 7 $\mathrm{mV}$ achieved. Even though the generated potential is not large enough to power a nanodevice but this approach provides a convenient way in the future to utilize the piezoelectric properties of $\mathrm{ZnO}$ nanowires grow on paper substrate with large enough power for different nanoscale devices.

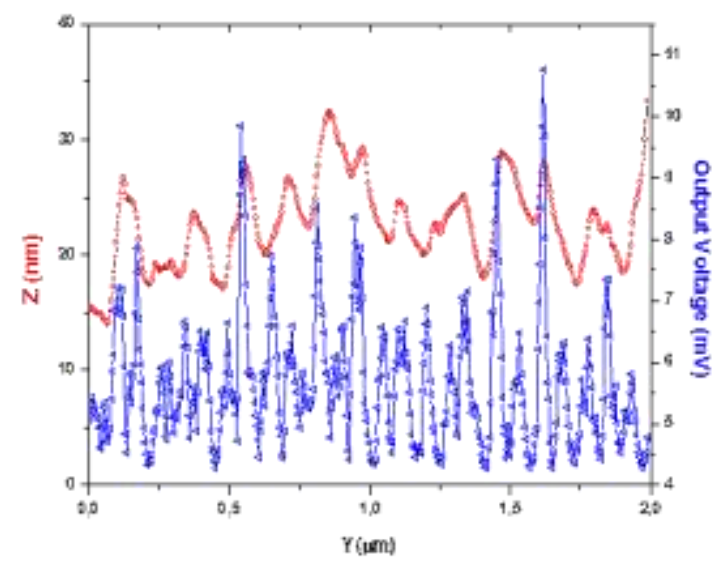

Figure 4 Typical tip scan signal profile of $\mathrm{ZnO}$ NWs from the AFM topography and the corresponding output voltage. The red circles show scanning and the blue triangles show the resulting output voltage.

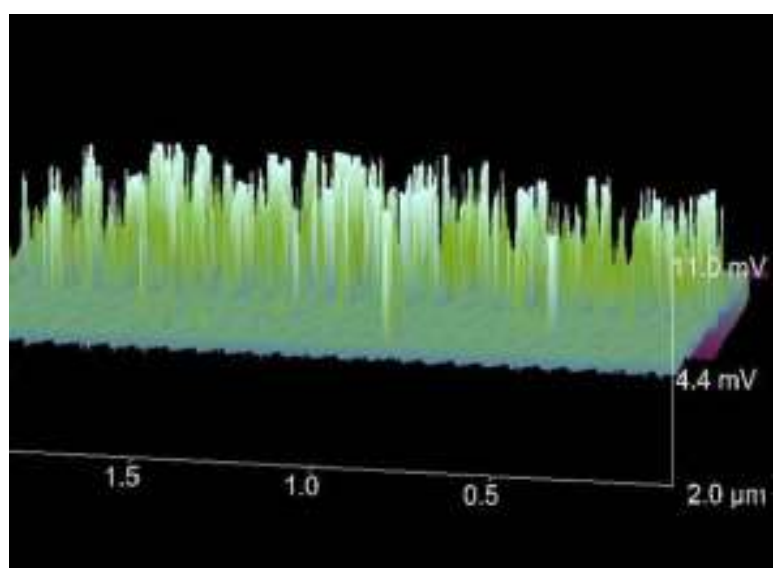

Figure 5 Three-dimensional plot of the output voltage at an external load records when the AFM tip scanned across the NWs array.
There are several reasons for observing low output piezo-potential e.g. density of states, surface morphology, structural differences and screening of ions by free carriers.

$\mathrm{ZnO} \mathrm{NWs}$ grown by the ACG method have higher density of states (DOS) due to the higher intrinsic doping concentration. The aspect ratio and the density of the $\mathrm{ZnO}$ NWs can also influence the output signals because the potential produced at the surface of the NWs is directly proportional to the lateral displacement and inversely proportional to the cubic square of the aspect ratio of the NWs [13]. Higher output voltage obtained when the deflection of the $\mathrm{ZnO} \mathrm{NWs}$ is maximum. Through the ACG method we obtained $\mathrm{ZnO}$ NWs with higher density, smaller length and larger diameter so the AFM tip may produce less deformation resulting in a relatively low output potential.

In summary, we have demonstrated piezoelectric power generation from $\mathrm{ZnO}$ nanowires grown on paper substrate by the low temperature ACG method. When the AFM deflects the $\mathrm{ZnO}$ NWs an output voltage of up to $7 \mathrm{mV}$ was observed. Furthermore the different factors which influence the magnitude of the output voltage have been discussed. This approach could be beneficial in power nanogenerators as it is highly effective and viable with several special properties like low-cost, flexible and fully recyclable process. This can provide a highly effective platform for powering different nanodevice like MEMS, NEMS and other devices.

\section{References}

[1] Z. L. Wang et al., Science 312, 242 (2006).

[2] Z. Fan et al., J. Nanoscience \& Nanotechnology, 5, 1561 (2005).

[3] R. F. Service, Science 328, 304 (2010).

[4] S.C. Minne et al., Appl. Phys. Lett. 67, 3918 (1995).

[5] J. R. Choi et al., J. Micromech. Microeng. 3, 60 (1993).

[6] K. Uchino, Smart Mater. Struct. 7, 273 (1998).

[7] Ü. Özgür et al., J. Appl. Phys. 98, 041301 (2005).

[8] Z. L. Wang et al., Nano Lett. 9, 3435 (2009).

[9] Z. L. Wang et al., Adv. Mater. 19, 781 (2007).

[10] Z. L. Wang et al., Nano Lett. 8, 2725 (2008).

[11] L.Vayssieres et al., Chem. Mater. 13, 233 (2001).

[12] M. Riaz et al., Adv. Fun. Mater. 20, 1 (2010).

[13] Z. L.Wang et al., Adv. Fun. Mater. 18, 3553 (2008). 